\title{
After the Fort McMurray wildfire there are significant increases in mental health symptoms in grade $7-12$ students compared to controls
}

Matthew R. G. Brown ${ }^{1,2}$, Vincent Agyapong ${ }^{2}$, Andrew J. Greenshaw², Ivor Cribben³, Pamela Brett-MacLean², Julie Drolet ${ }^{4}$, Caroline McDonald-Harker ${ }^{5}$, Joy Omeje ${ }^{2}$, Monica Mankowsi ${ }^{6}$, Shannon Noble ${ }^{7}$, Deborah Kitching ${ }^{6}$ and Peter H. Silverstone $2^{2^{*}}$

\begin{abstract}
Background: In order to examine the impact of disasters on adolescent mental health, this study compared population mental health survey data from two communities in Alberta, Canada: Fort McMurray, which experienced a major natural disaster, and Red Deer, which did not.

Methods: Data from 3070 grade 7-12 students from Fort McMurray, Alberta, Canada (collected in 2017, 18 months after the 2016 wildfire) was compared with data from 2796 grade 7-12 students from Red Deer, Alberta, Canada (collected in 2014). The same measurement scales were used for both surveys. Both of these cities have populations of approximately 100,000, and both cities are located in Alberta, Canada. For this reason, Red Deer is an appropriate non-disaster impacted community to compare to the disaster impacted community of Fort McMurray.

Results: The results of this comparison demonstrate that mental health symptoms were statistically significantly elevated in the Fort McMurray population when compared to the control population in Red Deer. This occurred for scores consistent with a diagnosis of depression (31\% vs. $17 \%$ ), moderately severe depression (17\% vs. $9 \%$ ), suicidal thinking (16\% vs. $4 \%)$, and tobacco use (13\% vs. $10 \%)$. Consistent with there being major mental health impacts from the 2016 Fort McMurray wildfire, self-esteem scores and quality of life scores were also statistically significantly lower in Fort McMurray. While the rates of anxiety disorder were similar (15\% vs. 16\%), the mean scores on the anxiety scale were slightly higher, with this difference reaching statistical significance. There were no statistical differences in the rates or scores for alcohol or substance use.

Conclusions: Our results are consistent with previous findings showing a significant negative impact of disasters on many aspects of adolescent mental, with a particular increase in symptoms related to depression and suicidal thinking. These findings highlight first, the need to identify adolescents most at risk of developing psychiatric symptoms after experiencing the trauma of disaster and second, the importance and necessity of implementing short and long term mental health intervention programs specifically aimed at adolescents, in order to help mitigate the negative effects of disasters on their mental health.
\end{abstract}

Keywords: Youth, Mental health, Wildfire, Disaster, Depression, Anxiety, PTSD, Substance use, Self-esteem, Resilience

\footnotetext{
* Correspondence: peter.silverstone@ualberta.ca

${ }^{2}$ Department of Psychiatry, University of Alberta, Edmonton, AB T6G 2B7,

Canada

Full list of author information is available at the end of the article
}

C) The Author(s). 2019, corrected publication March/2019. Open Access This article is distributed under the terms of the Creative Commons Attribution 4.0 International License (http://creativecommons.org/licenses/by/4.0/), which permits

unrestricted use, distribution, and reproduction in any medium, provided you give appropriate credit to the original author(s) and the source, provide a link to the Creative Commons license, and indicate if changes were made. The Creative Commons Public Domain Dedication waiver (http://creativecommons.org/publicdomain/zero/1.0/) applies to the data made available in this article, unless otherwise stated. 


\section{Background}

On May 3, 2016, the population of 88,000 living in Fort McMurray, Alberta, Canada was evacuated due to a major wildfire. The fire, dubbed "The Beast" in the popular media [1], destroyed 10\% of the homes in Fort McMurray and spread across 590,000 ha of land before being contained. A phased re-entry of residents began in early June 2016. According to the Insurance Bureau of Canada, the cost of the Fort McMurray wildfire was estimated at CAD $\$ 3.6$ billion, the most expensive insured catastrophe in Canadian history [2]. Extensive damage was caused to local infrastructures including homes, schools, and local businesses. Today, just two years post-wildfire, many individuals continue to be impacted by not only the physical damage caused to the community but also the social, emotional, and psychological difficulties that often occur in the aftermath of disaster [3]. Adolescents are particularly affected by disasters because of their dependence on adults and physical, psychological, and social factors related to their developmental stage. However, only a small number of studies have investigated the effects of natural disaster on adolescent mental health and well-being, either by comparing population mental health in disaster impacted versus non-disaster impacted communities or by comparing members of a disaster impacted community with differing levels of traumatic exposure. Previous studies suggest that wildfires have a negative impact on the mental health of residents of the affected areas (see [4] for review). Wildfires are unique in that their effects persist for an extended duration of time, thereby furthering the disruption of day-to-day functioning and ultimately often leading to reduced psychological adjustments and overall well-being $[5,6]$. After wildfires, children and adolescents exhibit increased incidence of post-traumatic stress disorder (PTSD) and depression [7, 8]. Studies conducted with both adults and children/adolescents report that both have an increased incidence of PTSD [9] as well as increased symptoms of depression and stress [10]. Studies focusing only on adults show that they exhibit increased symptoms of depression and anxiety [11, 12], increased incidence of PTSD $[11,13]$, increased levels of psychological distress [14], and increased consumption of anxiolyticshypnotics [15].

More broadly, non-wildfire disasters, such as floods, earthquakes, and tsunamis, also have a negative impact on mental health, as reviewed in Goldmann and Galea [16], Kar [17], and Norris et al. [18, 19]. Briefly, disasters are associated with increased incidence of major depressive disorder, generalized anxiety disorder, PTSD, and substance use disorder in children and adults. A link between disasters and increased depression is also supported by Tang et al. [20]. One more recent study associates earthquakes with increased incidence of PTSD [21]. Moreover, child and adolescent pre-existing mental health challenges are often exacerbated following exposure to disaster [22]. This is particularly concerning given that individuals', particularly adolescents', coping efficacy is an important mediating factor in the post-disaster recovery process and can have a significant impact on long-term mental health outcomes (also see Weems and Graham [23] on differential PTSD symptom recovery trajectories in adolescents exposed to hurricanes).

Using data from a battery of self-report questionnaires, the current study investigated population mental health effects in the form of depression, suicidal thinking, anxiety, alcohol/substance use disorder, tobacco use, self-esteem, and quality of life in the population of grade 7-12 students in Fort McMurray, Alberta following the wildfire. This data was compared to a sample of grade 7-12 students from Red Deer, Alberta who participated in a previously-conducted study "Empowering a Multimodal Pathway Towards Healthy Youth" (EMPATHY) in 2014. The EMPATHY study included the same mental health questionnaires [24, 25]. The Red Deer, Alberta student population served as the non-disaster impacted control group. Red Deer provides a good comparison to Fort McMurray as both cities are located in Alberta, Canada (Central and Northern Alberta, respectively); both have similar socioeconomic distributions; and both have populations of approximately 100,000 (Fort McMurray had a population of 88,000 in May 2016; Red Deer had a population of 98,600 in 2014 at the time of the EMPATHY study).

The current work investigates the effects of wildfire on mental health in adolescents with a sample size $(n=3070$ from Fort McMurray; $n=2976$ from Red Deer) that is larger than in any previous study, to our knowledge. This paper adds to a small but important literature on the impact of wildfires and other disasters on adolescent mental health, with implications for policy and service delivery in the aftermath of the 2016 Fort McMurray wildfire and more generally.

Based on previous research, our hypotheses were that students in the post-wildfire community of Fort McMurray would exhibit elevated symptoms of depression, suicidal thinking, anxiety, alcohol/substance misuse, and tobacco use, in addition to lower scores for self-esteem and quality of life in comparison to Red Deer.

\section{Methods}

\section{Overview and ethical considerations}

The data from Fort McMurray was collected in November 2017, 18 months after the 2016 wildfire. The two school boards in Fort McMurray - Fort McMurray Public Schools and Fort McMurray Catholic Schools - surveyed 3252 students in grades 7-12 in the community in order to evaluate the effectiveness of the school mental health and support programs put in place to assist students after 
the wildfire. The survey consisted of a battery of ten questionnaires assessing aspects of demographics, mental health, resilience, and the impact of the 2016 wildfire (details below). Researchers from the University of Alberta were asked to collaborate and provide assistance in designing the survey questionnaires and analyzing the survey data.

All survey data was collected under the auspices and ethical guidelines of the two Fort McMurray school systems. The survey was administered as part of their standard curriculum and as an ongoing assessment of the educational and support programs they had put in place after the wildfire (see Additional file 1: Appendix for list of programs). The selection of measurement instruments was determined by the school systems and was informed by the existing scholarly literature and findings. Parents and guardians were notified of the process by written letter two weeks prior to the administration of the survey in the schools, and they had the option to opt their child(ren) out of the survey. Students had the option to participate or not in the survey, and this was explained at the start of each survey data collection session (see details below). Survey participation was anonymous; participants were not asked for their names. After the data was collected, the anonymized data was made available for analysis by researchers from the University of Alberta. The analysis of the survey data was approved by the University of Alberta's Health Research Ethics Board on June 26th, 2017 (ethics protocol number Pro00072669).

The data from Red Deer was collected through the previous EMPATHY project, which surveyed population mental health indicators in public schools in Red Deer using seven questionnaires. Six of the seven questionnaires used in Red Deer were also used in Fort McMurray. The seventh questionnaire used in Red Deer was a demographics questionnaire. The Fort McMurray survey also included a demographics questionnaire, which was somewhat different from the Red Deer one. Full details of the EMPATHY project are available [24, 25]. In this paper, we focus on the six questionnaires that were used in both the communities of Fort McMurray and Red Deer, using Red Deer as a control population. Ethics considerations for data collection in the Red Deer EMPATHY project have been described in detail previously [24, 25] but were similar to those for the Fort McMurray data collection. The EMPATHY program itself was approved by the Health Research Ethics Committee of the University of Alberta on December 5th, 2013, ethics protocol number Pro00041063.

This paper provides a comparison and analysis of the survey data collected from grade 7-12 students in the disaster impacted community of Fort McMurray and the non-disaster impacted community of Red Deer, in order to examine the effects of disasters on the mental health of adolescents.

\section{Survey questionnaires}

Questionnaire details are shown in Table 1. Both the Fort McMurray and Red Deer surveys included a questionnaire on demographics, though the precise questions differed between the two sites (see Table 1 for details).

The surveys in both Fort McMurray and Red Deer shared the following six questionnaires:

1. The Patient Health Questionnaire, Adolescent version (PHQ-A) assesses symptoms of depression and suicidality $[26,27]$. This questionnaire provides a score for depression symptom severity from 0 to 36. In this scale, there were 11 questions in the Fort McMurray version compared to 13 questions in the Red Deer EMPATHY version. This is because the Fort McMurray and Red Deer surveys used slightly different versions of the PHQ-A (see details in Table 1). In particular, question \#9 in the Fort McMurray version was split into two questions (\#9 and 10) in the Red Deer version. To enable use of the same analytical procedure for both sites, we averaged the answers to the Red Deer PHQ-A questions \#9 and 10 and rounded to the nearest integer to derive an imputed answer to the Fort McMurray version's question \#9. Question \#11 in the Red Deer version of the PHQ-A was not present in the Fort McMurray version, and question \#11 in the Red Deer version was, therefore, not included in any of this paper's analyses.

2. The Hospital Anxiety and Depression Scale (HADS, 7 questions, anxiety-related questions only) assesses symptoms of anxiety [28]. This questionnaire provides a score for anxiety symptom severity from 0 to 21 .

3. The CRAFFT Questionnaire (CRAFFT, 9 questions) assesses symptoms of alcohol and substance misuse $[29,30]$. This questionnaire provides a score of alcohol / substance misuse severity from 0 to 6 .

4. Tobacco Use Questionnaire (2 questions) includes two questions on tobacco use: "Over the past month: Do you smoke tobacco products? Do you use smokeless tobacco products?" Note that the Red Deer version asks the two questions "Over the past 12 months" as opposed to past month.

5. The Rosenberg Self-Esteem Scale (Rosenberg, 10 questions) assesses self-esteem [31]. This questionnaire provides a self-esteem score from 0 to 30 .

6. The Kidscreen Questionnaire (Kidscreen-10, 11 questions) assesses quality of life [32]. This questionnaire provides a quality of life score from 0 to 44 . 
Table 1 - Questionnaire details

\begin{tabular}{cll}
\hline \multicolumn{2}{c}{ Questions } & Answer choices \\
\hline Ft McMurray Demographics Questionnaire & \\
1 & Are you at school right now, while you are taking the survey? & Yes, no \\
2 & Are you a student? & Yes, no \\
3 & What gender do you identify with? & Female, male, other, prefer not to say \\
4 & What is your age in years? & 10 years or less, 11 years, 12 years, 13 years, 14 years, 15 years, \\
5 & What is your school? & 16 years, 17 years, 18 years, 19 years, 20 years or more \\
6 & What grade are you in? & $7,8,9,10,11,12$, other \\
7 & What school were you in for grade 6? & Select from a list of all Ft McMurray schools with any classes \\
Red Deer EMPATHY Demographics Questionnaire & in grades 7-12 \\
1 & What gender do you identify with? & Select from a list of all Ft McMurray schools with grade 6 \\
2 & What is today's date? & \\
3 & What is your date of birth? & Female, male \\
4 & What grade are you in? & Date selection
\end{tabular}

Not at all, Several days, More than half the days, Nearly every day Same as above

Same as above

Same as above

Same as above

Same as above

Same as above

Same as above noticed. Or the opposite-being so figety or restless that you have been moving around a lot more than usual

$9 \quad$ Thoughts that you would be better off dead, or of hurting yourself in some way

Questions 10 and 11 asked only if answer to question 9 is not

"Not at all"

10 Has there been a time in the past month when you have had serious thoughts about ending your life?

11 Have you ever, in your WHOLE LIFE, tried to kill yourself or made a suicide attempt?

Red Deer EMPATHY Patient Health Questionnaire (PHQ-A, Depression Symptoms)

Over the past 2 weeks, how often have you been bothered by any of the following problems?

$1 \quad$ Feeling down, depressed, irritable or hopeless

Not at all, Several days, More than half the days, Nearly every day Little interest or pleasure in doing things?

Trouble falling or staying asleep, or sleeping too much

Poor appetite, weight loss, or overeating?

Feeling tired, or having little energy?

Feeling bad about yourself-or that you are a failure or that you have let yourself or your family down

Not at all, Several days, More than half the days, Nearly every day Not at all, Several days, More than half the days, Nearly every day Not at all, Several days, More than half the days, Nearly every day Not at all, Several days, More than half the days, Nearly every day Not at all, Several days, More than half the days, Nearly every day 
Table 1

\begin{tabular}{|c|c|c|}
\hline & Questions & Answer choices \\
\hline 7 & $\begin{array}{l}\text { Trouble concentrating on things, such as school work, reading } \\
\text { or watching television }\end{array}$ & Not at all, Several days, More than half the days, Nearly every day \\
\hline 8 & $\begin{array}{l}\text { Moving or speaking so slowly that other people could have } \\
\text { noticed. Or the opposite-being so figety or restless that you } \\
\text { have been moving around a lot more than usual }\end{array}$ & Not at all, Several days, More than half the days, Nearly every day \\
\hline 9 & Thought of hurting yourself in some way & Not at all, Several days, More than half the days, Nearly every day \\
\hline 10 & Thoughts that you would be better off dead & Not at all, Several days, More than half the days, Nearly every day \\
\hline \multirow[t]{2}{*}{11} & $\begin{array}{l}\text { If you checked off "any problems", how difficult have these } \\
\text { problems made it for you to do your work, take care of things } \\
\text { at home, or get along with other people? }\end{array}$ & $\begin{array}{l}\text { Not difficult at all; Somewhat difficult; Very difficult; Extremely } \\
\text { difficult }\end{array}$ \\
\hline & $\begin{array}{l}\text { Questions } 12 \text { and } 13 \text { asked only if answer to question } 10 \text { is not } \\
\text { "Not at all" }\end{array}$ & Yes, no \\
\hline 12 & $\begin{array}{l}\text { Has there been a time in the past month when you have had } \\
\text { serious thoughts about ending your life? }\end{array}$ & Yes, no \\
\hline 13 & Have you ever, in your WHOLE LIFE, tried to kill yourself or & Yes, no \\
\hline
\end{tabular}

Most of the time; A lot of the time; From time to time, occasionally; Not at all

Very definitely and quite badly; Yes, but not too badly; A little, but it doesn't worry me; Not at all

A great deal of the time; A lot of the time; From time to time, but not too often; Only occasionally

Definitely; Usually; Not often; Not at all

Not at all; Occasionally; Quite often; Very often

Very much indeed; Quite a lot; Not very much; Not at all

Very often indeed; Quite often; Not very often; Not at all

CRAFFT Questionnaire (Drugs/Alcohol/Tabacco)

During the past 12 months, did you:

$1 \quad$ Drink any alcohol (more than a few sips)?

Yes, no

Yes, no

Yes, no

Yes, no

yourself) who was "high" or had been using alcohol or drugs?

Questions 5-9 asked only if "yes" to one or more of questions $1-3$.

5 Do you ever use alcohol or drugs to RELAX, feel better about yourself, or fit in?

6 Do you ever use alcohol or drugs while you are by yourself, or ALONE?

7 Do you every FORGET things you did while using alcohol or drugs?

8 Do your FAMILY or FRIENDS ever tell you that you should cut down on your drinking or drug use?

$9 \quad$ Have you ever gotten into TROUBLE while you were using alcohol or drugs?

Yes, no

Yes, no

Yes, no

Yes, no

Yes, no

Tobacco Use Questionnaire

During the past month: 
Table 1 - Questionnaire details (Continued)

\begin{tabular}{|c|c|c|}
\hline & Questions & Answer choices \\
\hline 1 & Do you smoke tobacco products? & Yes, no \\
\hline 2 & Do you use smokeless tobacco products? & Yes, no \\
\hline \multicolumn{3}{|c|}{ Rosenberg Self-Esteem Scale } \\
\hline 1 & On the whole, I am satisfied with myself. & Strongly agree, Agree, Disagree, Strongly disagree \\
\hline 2 & At times, I think I am no good at all. & Strongly agree, Agree, Disagree, Strongly disagree \\
\hline 3 & I feel that I have a number of good qualities. & Strongly agree, Agree, Disagree, Strongly disagree \\
\hline 4 & I am able to do things as well as most other people & Strongly agree, Agree, Disagree, Strongly disagree \\
\hline 5 & I feel I do not have much to be proud of. & Strongly agree, Agree, Disagree, Strongly disagree \\
\hline 6 & I certainly feel useless at times. & Strongly agree, Agree, Disagree, Strongly disagree \\
\hline 7 & $\begin{array}{l}\text { I feel that I'm a person of worth, at least on an equal plane } \\
\text { with others. }\end{array}$ & Strongly agree, Agree, Disagree, Strongly disagree \\
\hline 8 & I wish I could have more respect for myself. & Strongly agree, Agree, Disagree, Strongly disagree \\
\hline 9 & All in all, I am inclined to feel that I am a failure. & Strongly agree, Agree, Disagree, Strongly disagree \\
\hline 10 & I take a positive attitude toward myself. & Strongly agree, Agree, Disagree, Strongly disagree \\
\hline \multicolumn{3}{|c|}{ Kidscreen Questionnaire (Quality of Life) } \\
\hline & Thinking about the last week: & \\
\hline 1 & Have you physically felt fit and well & Not at all, slightly, moderately, very, \\
\hline 2 & Have you felt full of energy? & Never, seldom, quite often, very often, \\
\hline 3 & Have you felt sad? & Never, seldom, quite often, very often, \\
\hline 4 & Have you felt lonely? & Never, seldom, quite often, very often, \\
\hline 5 & Have you had enough time for yourself? & Never, seldom, quite often, very often, \\
\hline 6 & $\begin{array}{l}\text { Have you been able to do the things that you want to do } \\
\text { in your free time? }\end{array}$ & Never, seldom, quite often, very often, always \\
\hline 7 & Have your parent(s) treated you fairly? & Never, seldom, quite often, very often, \\
\hline 8 & Have you had fun with your friends? & Never, seldom, quite often, very often, \\
\hline 9 & Have you got on well at school? & Not at all, slightly, moderately, very, \\
\hline 10 & Have you been able to pay attention? & Never, seldom, quite often, very often, \\
\hline 11 & In general, how would you say your health is? & Excellent, very good, good, fair, poor \\
\hline
\end{tabular}

The order of presentation of the six above questionnaires was randomized for each participant, in both the Red Deer and Fort McMurray surveys, to avoid possible sequence effects whereby earlier questionnaires might have influenced participants' answers to later questionnaires.

In addition, the Fort McMurray survey included the following three questionnaires, which are not analyzed here as they were not administered in the Red Deer EMPATHY project survey: The Impact of Fire Questionnaire (6 questions), a custom questionnaire assessing the impact of the 2016 wildfire on the participant; the Child PTSD Symptom Scale (CPSS, 19 questions [33]; and the Child and Youth Resilience Measure (CYRM-12, 12 questions) [34]. For the Fort McMurray survey, the three additional questionnaires were asked after the six questionnaires that were shared with the Red Deer survey, to prevent the additional questionnaires from affecting answers in any of the six shared questionnaires.
Thus, the shared questionnaires were identical between the two study sites except for minor differences in the demographics, PHQ-A, and tobacco use questionnaires, as noted above.

\section{Survey administration procedure}

In Fort McMurray, students participated in the survey using a laptop or desktop computer during regular school hours in almost all cases. Depending on the school, students either came to a computer laboratory or used laptops brought to their classroom. At the beginning of each survey session, the students were read a script (reproduced in the Additional file 1: Appendix) explaining the purpose of the survey, how to complete the survey, and guaranteeing participant confidentiality, anonymity, and voluntary participation. Students were also given an opportunity to ask questions before participating. Survey participation was anonymous, and the 
survey did not ask participants for their names. (A small number of students with special circumstances participated from home, using their own computers. These students were given a written version of the same script.) A total of 96 questions were asked in the survey battery used in Fort McMurray. Participation took most students less than $20 \mathrm{~min}$, with a small number of students taking as long as $50 \mathrm{~min}$. Participants were able skip questions, although in the script they were encouraged to answer all questions.

In Red Deer, students participated in the survey using a mobile app on tablets provided by the research team. Full details have been reported previously [24, 25].

\section{Cut-off scores and probable diagnoses}

It is recognized that any scores on a specific scale are not diagnostic. Previously-published cut-off points for probable diagnoses were utilized for the specific scales. These included measures of depression (from PHQ-A), anxiety (from HADS), and alcohol/substance use disorder (from CRAFFT). We use the term "probable diagnosis" because scores were based on self-report scales, not psychiatric clinical interviews. Nonetheless, existing literature reports substantial correspondence between psychiatric clinical diagnoses of depression, anxiety, PTSD, and alcohol/substance use disorder with probable diagnoses based on widely published cut-off scores for the above three sales $[29,30,35-37]$. Each of the three probable diagnoses was defined based on a threshold value for the appropriate scale. Thus, probable depression was defined as having a PHQ-A score of 11 or more [36]. Probable moderately severe depression was defined as having a PHQ-A score of 15 or more [35]. Probable anxiety was defined as having a HADS score of 11 or more [37]. Probable alcohol/substance use disorder was defined as having a CRAFFT score of 2 or more [29, 30]. Tobacco use was defined as answering "yes" to one or both of the two questions in the Tobacco Use Questionnaire. We also defined an "Any of 3 probable diagnoses" criterion as being positive for one or more of three probable diagnoses: depression, anxiety, alcohol/substance use disorder.

Suicidal thinking was defined, for Fort McMurray participants, as selecting the answer "Several days", "More than half the days", or "Nearly every day" to the 9th PHQ-A question: "Over the past 2 weeks, how often have you been bothered by any of the following problems? Thoughts that you would be better off dead, or of hurting yourself in some way?" as well as answering "Yes" to the 10th PHQ-A question: "Has there been a time in the past month when you have had serious thoughts about ending your life?" For Red Deer participants, suicidal thinking was defined as an imputed question \#9 answer of 1-3 (i.e. equivalent to "Several days", "More than half the days", or "Nearly every day") as well as answering "Yes" to the question "Has there been a time in the past month when you have had serious thoughts about ending your life?". See Survey Questionnaires section above for differences in PHQ-A between Fort McMurray and Red Deer as well as imputation of answer to question \#9 for Red Deer PHQ-A data.

PTSD symptom data from the CPSS was available only for the Fort McMurray students. We took a CPSS score of 15 or more as indicating probable diagnosis of PTSD [38].

\section{Statistical analysis}

We compared participants from Fort McMurray vs. Red Deer on each of the following 12 measures including 1) mean PHQ-A score, 2) mean HADS score, 3) mean CRAFFT score, 4) mean Rosenberg score, 5) mean Kidscreen score, 6) percent probable depression, 7) percent probable moderately severe depression, 8) percent suicidality thinking, 9) percent probable anxiety, 10) percent probable alcohol/substance use disorder, 11) percent tobacco use, and 12) percent any of three probable diagnoses (probable depression, anxiety disorder, or alcohol/ substance use disorder). Details of questionnaires and probable diagnoses are given above. For a given measure (eg: mean PHQ-A), only those participants who fully answered each specific relevant questionnaire or scale without skipping any questions were included in the analysis.

We used permutation testing for all statistical comparisons (\#iterations $=10^{5}$ ). Permutation testing is nonparametric and was chosen for its robustness against non-normality. All tests were two-sided two-sample tests, with a null hypothesis of no difference between the means of the two groups for the given test. In total, we performed 12 individual statistical tests. Multiple comparisons were addressed using the Benjamini-Hochberg method for false discovery rate (FDR) correction. FDR correction indicated a correction threshold of $p=0.021$. All analyses were done using in-house code built in the Clojure programming language (http://clojure.org).

Effect sizes reported in tables are Cohen's d (mean difference divided by pooled standard deviation).

There was a small but statistically significant difference in mean age between the Fort McMurray and Red Deer participants (see Demographics section in Results for full details). To exclude possible effects of age on the comparisons of interest, we re-ran all of the comparisons on a subset of the data, subsampled so as to make the distributions of ages as similar as possible between the subsampled Fort McMurray and Red Deer groups (including no significant difference in mean age). This subsampling did not qualitatively change the significant results. In addition, we re-ran all of the comparisons using the full sample of participants, after subtracting out participant age from the measure of 
interest. This procedure also did not qualitatively affect the significant results.

\section{Results}

The survey in Fort McMurray was administered to the entire population of grade 7-12 students in Fort McMurray. Five Public schools and two Catholics schools were involved in the survey. A total of 3252 students participated out of 4407 total students enrolled across both the Public and Catholic systems, i.e. a total of $72 \%$ of enrolled students participated in the survey. The survey data from the EMPATHY project in Red Deer was collected from 3244 participants at baseline (February and March 2014) from three middle schools (serving grades 6-8) and two high schools (serving grades 9-12) in the Red Deer Public School System. For full details of the EMPATHY project, see Silverstone et al. [24, 25]. The primary causes of non-participation were student absences from school during the surveys and logistics constraints. Though Red Deer and Fort McMurray schools devoted substantial resources and staff time to survey data collection, the surveys were done during regular class time, and it was logistically impossible to survey some students due to scheduling conflicts, exams, and so on. Fewer than 50 students in each city chose to decline participation in the survey.

\section{Data exclusion}

Of the 3252 students who participated in the Fort McMurray survey, we excluded 182 because of one or more of the exclusion criteria below. We excluded 448 of the 3244 participants from the Red Deer EMPATHY project, mostly as the majority of the excluded Red Deer participants were in grade 6 and the Fort McMurray survey did not include grade 6 students. Exclusion criteria were as follows:

1. In grade 6

2. Age $<=10$ years

3. Age $>=20$ years

4. Inconsistent answers among the positive and negative questions from the Rosenberg questionnaire (details in Additional file 1: Appendix 5).

5. Inconsistent answers among the positive questions from the Rosenberg questionnaire and the positive questions from the Kidscreen questionnaire (details in Additional file 1: Appendix 5).

6. Inconsistent answers among the non-reversed and reversed questions from the HADS questionnaire (answer order for two HADS questions is reversed to test for consistency, details in Additional file 1: Appendix 5).
Criteria 4 through 6 above were designed to exclude participants who gave inconsistent answers, possibly because they were not paying attention to the survey or did not understand the questions. After exclusions, there were 3070 participants remaining in the Fort McMurray dataset and 2796 participants remaining in the Red Deer EMPATHY dataset. It is these two datasets that were used for statistical analysis.

\section{Demographics}

Demographics for the 3070 Fort McMurray participants used for statistical analysis were as follows: gender identification was $48 \%$ female, $48 \%$ male, $2 \%$ other, and $2 \%$ preferred not to say, and age ranged from 11 to 19 , with a mean age of 14.3 and standard deviation 1.8. Demographics for the 2796 Red Deer EMPATHY project participants used for statistical analysis were as follows: gender identification was $48 \%$ female and $52 \%$ male, and age ranged from 11 to 19 , with mean 14.8 and standard deviation 1.7. Means ages for the Fort McMurray and Red Deer students were significantly different $(p=$ 0.00001 , permutation test, $10^{5}$ iterations). We ran additional analyses to exclude the possibility that this difference in mean age might have affected the comparisons of interest, and it did not (see end of Statistical Analyses section in the Methods).

\section{Mental health Indicator comparisons}

Comparison of the participant data from Fort McMurray (post-disaster) and Red Deer (no disaster) revealed significant differences in 8 of the 12 measures tested (see Table 2). PHQ-A depression scores were significantly higher in Fort McMurray, as were rates of probable depression, probable moderately severe depression, suicidal thinking, and tobacco use. Rosenberg self-esteem scores and Kidscreen quality of life scores were significantly lower in Fort McMurray. HADS anxiety scores were significantly higher in Fort McMurray, but rates of probable anxiety were not significantly different between the two cities. CRAFFT alcohol / substance misuse scores were also not significantly different, nor were rates of probable alcohol/substance use disorder. In addition, rates of "any of 3 probable diagnoses" were not significantly different. Each comparison included only data from participants who completed all questions in the relevant questionnaires. Numbers of students ranged from 2970 to 3070 in the Fort McMurray group, in comparison to 2796 (consistent across all questionnaires) in the Red Deer group.

PTSD symptom data from the CPSS questionnaire was available only for the Fort McMurray group. For that group, CPSS scores had a mean of 12.8 with standard deviation 11.5. Among Fort McMurray students, 37\% met conditions for probable diagnosis of PTSD based on 
Table 2 - Fort McMurray survey post-wildfire data vs. Red Deer EMPATHY survey control data

\begin{tabular}{|c|c|c|c|c|}
\hline Measure & Fort McMurray Score & Red Deer Score & $P$-value & Effect size \\
\hline PHQ-A score & $8.0+/-6.5$ & $5.6+/-5.6$ & $0.00001^{*}$ & 0.40 \\
\hline HADS score & $7.7+/-4.7$ & $7.4+/-5.0$ & $0.021^{*}$ & 0.06 \\
\hline CRAFFT score & $0.55+/-1.25$ & $0.54+/-1.16$ & 0.75 & 0.01 \\
\hline Rosenberg score & $18.2+/-6.6$ & $20.5+/-5.9$ & $0.00001^{*}$ & -0.36 \\
\hline Kidscreen score & $27.0+/-8.2$ & $29.4+/-7.3$ & $0.00001^{*}$ & -0.32 \\
\hline Measure & Rate & Rate & $P$-value & \\
\hline Probable depression & $31 \%$ & $17 \%$ & $0.00001^{*}$ & \\
\hline Probable moderately severe depression & $17 \%$ & $9 \%$ & $0.00001^{*}$ & \\
\hline Suicidal thinking & $16 \%$ & $4 \%$ & $0.00001^{*}$ & \\
\hline Probable anxiety & $27 \%$ & $27 \%$ & 0.98 & \\
\hline Probable alcohol / substance use disorder & $15 \%$ & $16 \%$ & 0.29 & \\
\hline Tobacco use & $13 \%$ & $10 \%$ & $0.00090^{*}$ & \\
\hline Any of 3 probable diagnoses & $37 \%$ & $36 \%$ & 0.50 & \\
\hline
\end{tabular}

* $p$-value survives FDR multiple comparison correction (threshold 0.021) Scores are means +/- standard deviation (not standard error)

thresholding CPSS scores with a cutoff of 15 [38]. CPSS data were not collected for the Red Deer group.

\section{Discussion}

This study compared survey data collected from grade 7-12 students in the disaster impacted community of Fort McMurray and the non-disaster impacted community of Red Deer, in order to examine the effects of disasters on the mental health of adolescents. Consistent with our hypotheses, we observed higher mean PHQ-A scores for depression symptoms, rates of probable depression, suicidal thinking, and tobacco use among grade 7-12 students in the disaster impacted community of Fort McMurray in comparison to the non-disaster impacted community of Red Deer. Rosenberg self-esteem scores and Kidscreen quality of life scores were also lower in the Fort McMurray student population compared to the Red Deer student population. These findings suggest that the wildfire had significant adverse effects on the mental health of adolescents in Fort McMurray due to the traumatic nature of disasters. These results are consistent with the literature which reports a negative impact of wildfire disasters on mental health $[7,9,10,12-15,39-42]$. These findings emphasize the need for mental health policies, programs, and supports specifically targeted to adolescents following disaster in order to reduce their vulnerabilities and build positive mental health.

Rates of probable anxiety and probable alcohol/substance use disorder were very similar between Fort McMurray and Red Deer students, counter to our original hypotheses. This was unexpected as population mental health trends for depression tend to match those for anxiety and alcohol/substance use disorder [16]. Fort McMurray Public and Catholic Schools put in place substantial mental health support programming for students after the 2016 wildfire. (See Additional file 1: Appendix for list of mental health support programs.) These results are consistent with the effectiveness of the mental health supports in reducing rates of probable anxiety and alcohol/substance use disorder in the aftermath of the wildfire, though we do not have the pre-fire data necessary to fully support this argument quantitatively. Another possibility (not mutually exclusive) is that these findings may suggest that experiencing a disaster has less impact on adolescents' rates of probable anxiety and probable alcohol/substance use in the short term (given that the Fort McMurray data was collected just 18 months after the wildfire). The literature indicates that psychological impacts of disaster occur both in the short-term (immediately after evacuation and in the 2 to 5 years rebuilding phase), but also persist in the long-term (up to 10 years during the recovery phase) [43]. These findings highlight the need for a wide range of mental health supports for adolescents following disasters which address both current and potential long-term psychiatric symptoms, in order to support positive mental health among adolescents.

We believe that the Red Deer data provides a compelling community control group given the similarities between communities. Nonetheless, this data was not specifically collected with this intent. A possible issue could be that, while Red Deer experienced no natural disasters prior to or during the survey period, it is conceivable that the 3 year difference in the time of the surveys may have had a minor impact due to some other effect. However, the 2016 wildfire had such a large impact on Fort McMurray and the findings presented here are so striking that we believe any effect from the 3 year difference in survey times would be much smaller than the 
effects of the 2016 wildfire. In addition, it would have been useful to compare data for symptoms related to disaster trauma exposure between the Fort McMurray and Red Deer samples. This was not possible as we did not have such data from the Red Deer sample.

In conclusion, the current results support existing findings which indicate that adolescents are vulnerable to, and adversely impacted by disasters. The present data extends this by comparing the mental health of a large sample $(n=3070)$ of grade 7-12 students in the disaster impacted community of Fort McMurray to a similarly large sample $(n=2796)$ of adolescents in the non-disaster impacted community of Red Deer, identifying those in Fort McMurray as more vulnerable and at greater risk of low mental health. (To mitigate this, Fort McMurray Public and Catholic Schools have made significant and ongoing efforts to put in place mental health support programs for their students in the aftermath of the wildfire.) The results of this study highlight the importance of longer-term support for students impacted by disasters, particularly focused on depressive symptoms.

\section{Additional file}

Additional file 1: Appendix - Survey Description Script includes a copy of the script read to each class before survey data collection in Fort McMurray. Appendix - Details of Exclusion Criteria contains additional details of the exclusion criteria used for excluding some participants' data from the analysis, as described in the "Data Exclusion" section in the main text. Appendix - Mental Health Support Programs includes a list of mental health support programs put in place by the Fort McMurray Public and Catholic Schools after the 2016 wildfire. (DOCX $15 \mathrm{~kb}$ )

\section{Abbreviations}

CPSS: Child PTSD Symptom Scale; CRAFFT: CRAFFT Questionnaire (proper name of the questionnaire is (RAFFT); CYRM-12: Child and Youth Resilience Measure; EMPATHY: Empowering a Multimodal Pathway Towards Healthy Youth project; FDR: False discovery rate; HADS: Hospital Anxiety and Depression Scale; Kidscreen-10: Kidscreen Questionnaire; PHQ-A: The Patient Health Questionnaire, Adolescent version; PTSD: post-traumatic stress disorder; Rosenberg: Rosenberg Self-Esteem Scale

\section{Acknowledgements}

We thank Fort McMurray Public and Catholic Schools for allowing us to analyze an anonymized dataset arising from their survey. We also thank all individuals involved in the survey data collection including Fort McMurray teachers, counsellors, and other staff members. We thank the EMPATHY study research team and all the individuals who made the EMPATHY study possible.

\section{Funding}

Funding for support to the Fort McMurray schools in developing their survey and support for this subsequent statistical analysis were provided in part by collaborative grant funding from the Canadian Institutes of Health Research, Canadian Red Cross, and Alberta Innovates Health Solutions (grant number 201600546)

The EMPATHY study had significant institutional support in Alberta including that of the Science, Policy Practice Network, a multi-sectoral partnership supported in part by the Norlien Foundation of Alberta and grant funding from Alberta Health Services (Grant-002479).

\section{Availability of data and materials}

The datasets analysed in the current study are available from the corresponding author on reasonable request.

\section{Authors' contributions}

Study design: MB VA AG PB JD CM JO MM SN DK PS. Data collection: MM SN DK. Analysis: MB IC PS. Manuscript preparation: MB IC PB JD CM PS. Some portions of the manuscript, mostly in the Methods section, are shared with a separate manuscript in preparation by the authors presenting a distinct set of findings based on a separate analysis of the data from Fort McMurray. All authors read and approved the final manuscript.

\section{Ethics approval and consent to participate}

All survey data from Fort McMurray was collected under the auspices and ethical guidelines of the two Fort McMurray school systems and was administered as part of their standard curriculum and as an ongoing assessment of the educational and support programs they had put in place after the wildfire. Parents and guardians were notified of the process by written letter two weeks prior to the administration of the survey in the schools, and they had the option to opt their child(ren) out of the survey. Students had the option to participate or not in the survey, and this was explained at the start of each survey data collection session. Verbal informed consent was given by all participants. Survey participation was anonymous; participants were not asked for their names. After the data was collected, the anonymized data was made available for analysis by researchers from the University of Alberta. The analysis of the survey data was approved by the University of Alberta's Health Research Ethics Board on June 26th, 2017 (ethics protocol number Pro00072669).

The data from Red Deer was collected through the previous EMPATHY project. The EMPATHY program was approved by the Health Research Ethics Committee of the University of Alberta on December 5th, 2013 (ethics protocol number Pro00041063).

\section{Consent for publication}

Not applicable.

\section{Competing interests}

The authors declare that they have no competing interests in the presentation of these findings.

\section{Publisher's Note}

Springer Nature remains neutral with regard to jurisdictional claims in published maps and institutional affiliations.

\section{Author details}

'Department of Computing Science, University of Alberta, Edmonton, Canada. ${ }^{2}$ Department of Psychiatry, University of Alberta, Edmonton, AB T6G 2B7, Canada. ${ }^{3}$ Alberta School of Business, University of Alberta, Edmonton, Canada. ${ }^{4}$ Faculty of Social Work, University of Calgary, Calgary, Canada. ${ }^{5}$ Department of Sociology and Anthropology, Mount Royal University, Calgary, Canada. ${ }^{6}$ Fort McMurray Catholic School District, Fort McMurray, Canada. ${ }^{7}$ Fort McMurray Public School District, Fort McMurray, Canada.

Received: 23 May 2018 Accepted: 27 December 2018

Published online: 10 January 2019

\section{References}

1. CBC. Devastating Fort McMurray wildfire declared out 15 months later |CBC News. 2017. http://www.cbc.ca/news/canada/edmonton/fort-mcmurray-firebeast-extinguished-out-1.4271604. Accessed 17 Apr 2018.

2. Cryderman K. Fort McMurray wildfires to cost insurers $\$ 3.6$-billion - The Globe and Mail. 2016. https://www.theglobeandmail.com/report-onbusiness/fort-mcmurray-wildfire-damage-to-cost-36-billion-insurancebureau/article30788517/. Accessed 10 May 2018.

3. Cohan $\mathrm{CL}$, Cole SW. Life course transitions and natural disaster: marriage, birth, and divorce following hurricane Hugo. J Fam Psychol. 2002;16:14-25 http://www.ncbi.nlm.nih.gov/pubmed/11915406. Accessed 10 May 2018.

4. Laugharne J, Van de Watt G, Janca A, Janca A. After the fire: the mental health consequences of fire disasters. Curr Opin Psychiatry. 2011;24:72-7. https://doi.org/10.1097/YCO.0b013e32833f5e4e. 
5. McFarlane AC, Clayer JR, Bookless CL. Psychiatric morbidity following a natural disaster: an Australian bushfire. Soc Psychiatry Psychiatr Epidemiol. 1997;32:261-8 http://www.ncbi.nlm.nih.gov/pubmed/9257516. Accessed 17 May 2018.

6. Marshall GN, Schell TL, Elliott MN, Rayburn NR, Jaycox LH. Psychiatric disorders among adults seeking emergency disaster assistance after a wildland-urban Interface fire. Psychiatr Serv. 2007;58:509-14. https://doi.org/ 10.1176/ps.2007.58.4.509.

7. McDermott BM, Lee EM, Judd M, Gibbon P. Posttraumatic stress disorder and general psychopathology in children and adolescents following a wildfire disaster. Can J Psychiatr. 2005;50:137-43. https://doi.org/10.1177/ 070674370505000302.

8. Papadatou D, Giannopoulou I, Bitsakou P, Bellali T, Talias MA, Tselepi K Adolescents' reactions after a wildfire disaster in Greece. J Trauma Stress. 2012;25:57-63. https://doi.org/10.1002/jts.21656.

9. Jones RT, Ribbe DP, Cunningham PB, Weddle JD, Langley AK. Psychological impact of fire disaster on children and their parents. Behav Modif. 2002;26: 163-86. https://doi.org/10.1177/0145445502026002003.

10. Kirsch KR, Feldt BA, Zane DF, Haywood T, Jones RW, Horney JA. Longitudinal community assessment for public health emergency response to wildfire, Bastrop County, Texas. Heal Secur. 2016;14:93-104. https://doi. org/10.1089/hs.2015.0060.

11. Bryant RA, Gibbs L, Gallagher HC, Pattison P, Lusher D, MacDougall C, et al. Longitudinal study of changing psychological outcomes following the Victorian black Saturday bushfires. Aust New Zeal J Psychiatry. 2017: 000486741771433. https://doi.org/10.1177/0004867417714337.

12. Papanikolaou V, Adamis D, Mellon RC, Prodromitis G. Psychological distress following wildfires disaster in a rural part of Greece: a case-control population-based study. Int J Emerg Ment Health. 2011;13:11-26 http:// www.ncbi.nlm.nih.gov/pubmed/21957753. Accessed 12 Mar 2018.

13. Psarros C, Theleritis C, Economou M, Tzavara C, Kioulos KT, Mantonakis L, et al. Insomnia and PTSD one month after wildfires: evidence for an independent role of the "fear of imminent death". Int J Psychiatry Clin Pract. 2017;21:137-41. https://doi.org/10.1080/13651501.2016.1276192.

14. Afifi WA, Felix ED, Afifi TD. The impact of uncertainty and communal coping on mental health following natural disasters. Anxiety, Stress Coping. 2012; 25:329-47. https://doi.org/10.1080/10615806.2011.603048.

15. Caamano-Isorna F, Figueiras A, Sastre I, Montes-Martínez A, Taracido M, Piñeiro-Lamas M. Respiratory and mental health effects of wildfires: an ecological study in Galician municipalities (north-West Spain). Environ Health. 2011;10:48. https://doi.org/10.1186/1476-069X-10-48.

16. Goldmann E, Galea S. Mental health consequences of disasters. Annu Rev Public Health. 2014;35:169-83. https://doi.org/10.1146/annurev-publhealth032013-182435.

17. Kar N. Psychological impact of disasters on children: review of assessment and interventions. World J Pediatr. 2009;5:5-11. https://doi.org/10.1007/ s12519-009-0001-X

18. Norris FH, Friedman MJ, Watson PJ, Byrne CM, Diaz E, Kaniasty K. 60,000 disaster victims speak: Part I. An empirical review of the empirical literature, 1981-2001. Psychiatry. 2002;65:207-39 http://www.ncbi.nIm.nih.gov/ pubmed/12405079. Accessed 23 Apr 2018.

19. Norris FH, Friedman MJ, Watson PJ. 60,000 disaster victims speak: part II. Summary and implications of the disaster mental health research. Psychiatry. 2002;65:240-60 http://www.ncbi.nlm.nih.gov/pubmed/12405080. Accessed 23 Apr 2018

20. Tang B, Liu X, Liu Y, Xue C, Zhang L. A meta-analysis of risk factors for depression in adults and children after natural disasters. BMC Public Health. 2014;14:623. https://doi.org/10.1186/1471-2458-14-623.

21. Dai W, Chen L, Lai Z, Li Y, Wang J, Liu A. The incidence of post-traumatic stress disorder among survivors after earthquakes:a systematic review and meta-analysis. BMC Psychiatry. 2016;16:188. https://doi.org/10.1186/s12888016-0891-9.

22. Martin NC, Felton JW, Cole DA. Predictors of youths' posttraumatic stress symptoms following a natural disaster: the 2010 Nashville, Tennessee, flood. J Clin Child Adolesc Psychol. 2016;45:335-47. https://doi.org/10.1080/ 15374416.2014.982279.

23. Weems CF, Graham RA. Resilience and trajectories of posttraumatic stress among youth exposed to disaster. J Child Adolesc Psychopharmacol. 2014 24:2-8. https://doi.org/10.1089/cap.2013.0042.

24. Silverstone PH, Bercov M, Suen VYM, Allen A, Cribben I, Goodrick J, et al. Initial findings from a novel school-based program, EMPATHY, which may help reduce depression and suicidality in youth. PLoS One. 2015;10: e0125527. https://doi.org/10.1371/journal.pone.0125527.

25. Silverstone PH, Bercov M, Suen VYM, Allen A, Cribben I, Goodrick J, et al. Long-term results from the empowering a multimodal pathway toward healthy youth program, a multimodal school-based approach, show marked reductions in suicidality, depression, and anxiety in 6,227 students in grades 6-12 (aged 11-18). Front Psychiatry. 2017;8:81. https://doi.org/10.3389/fpsyt. 2017.00081.

26. Johnson JG, Harris ES, Spitzer RL, Williams JBW. The patient health questionnaire for adolescents: validation of an instrument for the assessment of mental disorders among adolescent primary care patients. J Adolesc Health. 2002;30:196-204 http://www.ncbi.n/m.nih.gov/pubmed/ 11869927. Accessed 5 Mar 2018.

27. Spitzer RL, Kroenke K, Williams JBW. Group and the PHQPCS. Validation and utility of a self-report version of PRIME-MD: the PHQ primary care study. JAMA. 1999;282:1737. https://doi.org/10.1001/jama.282.18.1737.

28. Zigmond AS, Snaith RP. The hospital anxiety and depression scale. Acta Psychiatr Scand. 1983;67:361-70 http://www.ncbi.nlm.nih.gov/pubmed/ 6880820. Accessed 5 Mar 2018.

29. Knight JR, Shrier LA, Bravender TD, Farrell M, Vander Bilt J, Shaffer HJ. A new brief screen for adolescent substance abuse. Arch Pediatr Adolesc Med. 1999;153:591-6 http://www.ncbi.nlm.nih.gov/pubmed/10357299. Accessed 5 Mar 2018.

30. Knight JR, Sherritt L, Harris SK, Gates EC, Chang G. Validity of brief alcohol screening tests among adolescents: a comparison of the AUDIT, POSIT, CAGE, and CRAFFT Alcohol. Clin Exp Res. 2003;27:67-73. https://doi.org/10. 1097/01.ALC.0000046598.59317.3A.

31. Rosenberg M. Society and the adolescent self-image. Princeton, NJ: Princeton University Press; 1965.

32. Ravens-Sieberer U, Erhart M, Rajmil L, Herdman M, Auquier P, Bruil J, et al. Reliability, construct and criterion validity of the KIDSCREEN-10 score: a short measure for children and adolescents' well-being and health-related quality of life. Qual Life Res. 2010;19:1487-500. https://doi.org/10.1007/ s11136-010-9706-5.

33. Foa EB, Johnson KM, Feeny NC, Treadwell KR. The child PTSD symptom scale: a preliminary examination of its psychometric properties. J Clin Child Psychol. 2001;30:376-84. https://doi.org/10.1207/\$15374424JCCP3003 9.

34. Liebenberg L, Ungar M, LeBlanc JC. The CYRM-12: a brief measure of resilience. Can J Public Health. 2013;104:e131-5 http://www.ncbi.nlm.nih. gov/pubmed/23618205. Accessed 5 Mar 2018.

35. Kroenke K, Spitzer RL, Williams JB. The PHQ-9: validity of a brief depression severity measure. J Gen Intern Med. 2001;16:606-13. https://doi.org/10.1046/ J.1525-1497.2001.016009606.X.

36. Richardson LP, McCauley E, Grossman DC, McCarty CA, Richards J, Russo JE, et al. Evaluation of the patient health Questionnaire-9 item for detecting major depression among adolescents. Pediatrics. 2010;126:1117-23. https:// doi.org/10.1542/peds.2010-0852.

37. Snaith RP. The hospital anxiety and depression scale. Health Qual Life Outcomes. 2003;1(29). https://doi.org/10.1186/1477-7525-1-29.

38. Stewart RW, Ebesutani C, Drescher CF, Young J. The child PTSD symptom scale: an investigation of its psychometric properties. J Interpers Violence. 2015;32:2237-56. https://doi.org/10.1177/0886260515596536.

39. Jones RT, Ribbe DP, Cunningham P. Psychosocial correlates of fire disaster among children and adolescents. J Trauma Stress. 1994;7:117-22 http:// www.ncbi.nlm.nih.gov/pubmed/8044435. Accessed 12 Mar 2018.

40. Tally S, Levack A, Sarkin AJ, Gilmer T, Groessl EJ. The impact of the San Diego wildfires on a general mental health population residing in evacuation areas. Adm Policy Ment Heal Ment Heal Serv Res. 2013;40:34854. https://doi.org/10.1007/s10488-012-0425-9.

41. Wasiak J, Mahar P, Lee S, Paul E, Spinks A, Pfitzer B, et al. 12-month generic health status and psychological distress outcomes following an Australian natural disaster experience: 2009 black Saturday wildfires. Injury. 2013;44: 1443-7. https://doi.org/10.1016/j.injury.2012.08.060.

42. Felix E, Afifi T, Kia-Keating M, Brown L, Afifi W, Reyes G. Family functioning and posttraumatic growth among parents and youth following wildfire disasters. Am J Orthop. 2015;85:191-200. https://doi.org/10.1037/ort0000054.

43. Salzer M, Bickman L. The short and long term psychological impact of disasters: implications for mental health interventions and policy. In: Gist R, Lubin B, editors. Response to disaster: psychosocial, community, and ecological approaches. In. Philadelphia: Brunner/Maze; 1999. p. 63-89. 